\title{
Deployment of Agent-Based Intrusion Detection for Wireless LAN
}

\author{
G.K.Viju' ${ }^{1}$, Khalid Ahmed Ibrahim ${ }^{2}$
}

\begin{abstract}
Security has always been a concern in communication networks as it is in many other areas. The important typical security issues that one should consider are threats to the physical network, unauthorized access to network resources and internal and external attacks. In the context of wired LANs, the solutions to the above issues are well defined and significantly reliable. The same approaches, however, cannot be adapted to the wireless LANs. In this paper, Agent-based intrusion detection for wireless LANs is proposed to detect unauthorized wireless elements like access points, wireless clients that are in promiscuous mode, etc. The system reacts to intrusions by either notifying the concerned personnel, in case of rogue access points and promiscuous nodes, or blocking unauthorized users from accessing the network resources.
\end{abstract}

Keywords: Wireless LANs, Intrusion Detection, Security, Rogue Access Point.

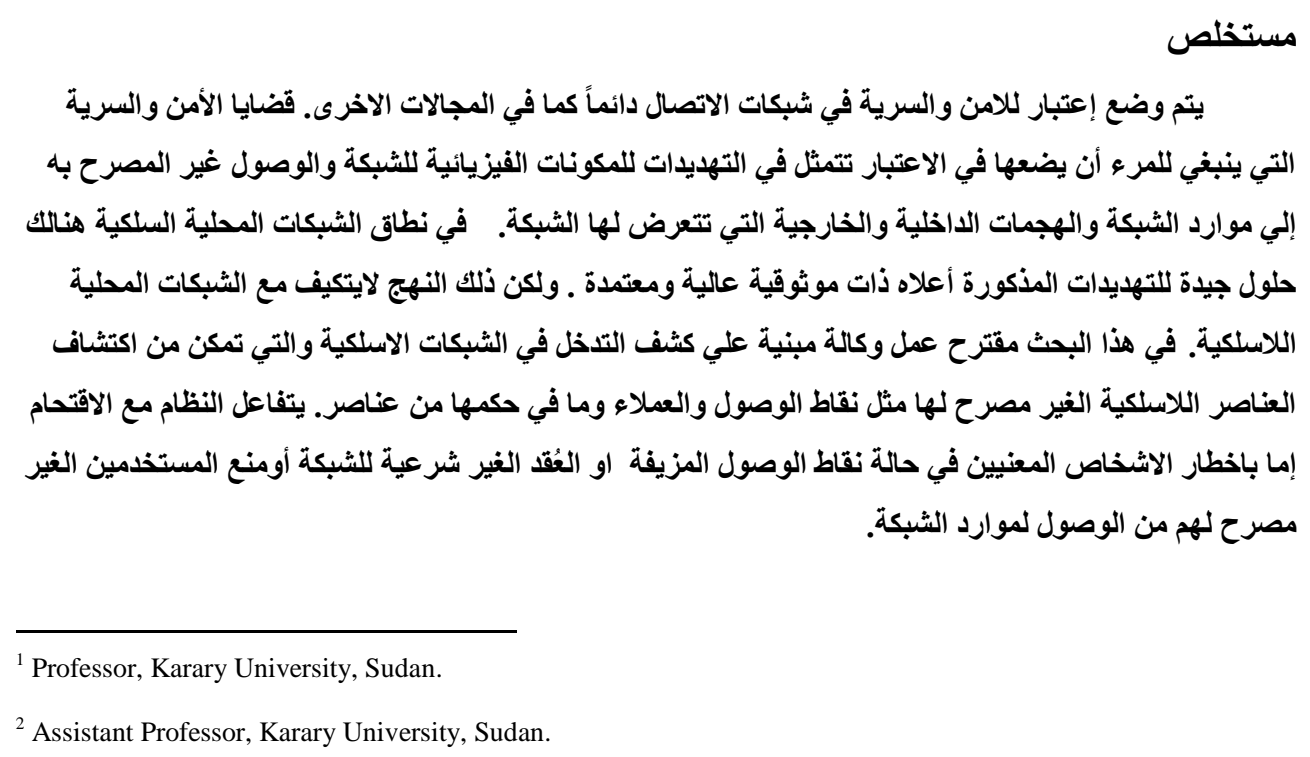




\section{Introduction}

Wireless Local Area Network technology, despite the numerous advantages it has over competing technologies, has not seen widespread deployment. A primary reason for markets not adopting this technology is its failure to provide adequate security. Data that is sent over wireless links can be compromised with utmost ease. In the context of wired LANs, the solutions to the above issues are well defined and significantly reliable. The important typical security issues include threats to the physical network, unauthorized access to network resources, and both internal and external attacks like Denial of Service (DoS).

\subsection{Security in the wireless network}

WEP (Wired Equivalent Privacy) is the security protocol provided in the IEEE 802.11 [8] wireless standard to bring the security in wireless networks comparable to the security in wired networks. Its main objective is to protect the data that is transmitted over wireless links from malicious eavesdroppers. WEP tries to accomplish its goal by encrypting the data that is being transmitted at the MAC layer. The main scheme that is being employed in WEP is shared key authentication. Shared key cryptography is used to authenticate and also to communicate with a mobile node. However, studies $[1,2,4]$ have proved WEP to be a complete failure. In [1] it has been shown that WEP can be compromised by successfully modifying data, injecting fake data and decrypting data. As a result it has been proved that Authentication, Authorization and Accounting (AAA) are all not achieved with WEP. 


\subsection{Intrusion into wireless LAN}

Intrusion, in the field of networks, is defined as the act of wrongfully accessing the network resources without having appropriate privileges. Intrusion into wireless networks is relatively easier when compared to wired networks. Passively sniffing the network is the easiest intrusion that one can attempt with just a wireless card and a laptop. The wireless card can be slipped into RFMODE (promiscuous mode) and all the data in the air can be sniffed. Intrusion of this kind is possible if and only if the network has wireless access points installed on the wired network. In the case where the network has inaccessible access points, one can intrude into a corporate network by gaining physical access to the wired network and installing a rogue access point. In either case, the attacks can either be internal - attack carried by people within the organization or external - attack carried by people outside the organization. In the latter type of intrusion, it is as simple as passively sniffing the network once the access point is installed.

\section{Materials and Methodology}

Agent-based intrusion detection system detects the presence of unauthorized wireless elements, namely, rogue access points, wireless promiscuous nodes and unauthorized clients. Depending on the kind of wireless element detected, the system would respond accordingly. In the case of the wireless element being a rogue access point or a wireless promiscuous node, the response would be sending the relevant information such as the geographical information, the time when the element was detected, etc. to the concerned personnel. If the wireless element turns out to 
be an unauthorized client, the response would be blocking that client from getting onto the network, thus preventing unauthorized access.

\subsection{Central Administrator}

The Central Administrator is the main central server that acts as the centralized entity to monitor all the wireless cells in the network. It maintains a record for each access point consisting of information about the geographical location it is serving, its MAC address, the name and IP address of the agent present in that cell, status of WEP etc. This information can help network administrators in making strategic decisions in strengthening the security of the network. The following are the functions offered by the central administrator:

1. Registering and unregistering access points (AP), client cards, and agents.

2. Scanning the network.

3. Generating x.509 certificates for client.

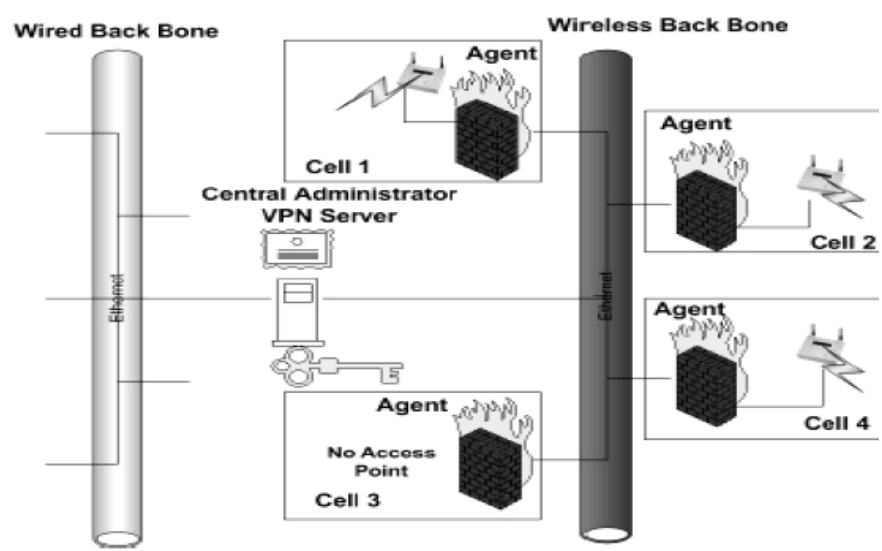

Figure (1): Overview of the Architecture 


\subsection{Agents}

Agents detect unauthorized wireless elements like rogue access points and promiscuous wireless nodes. Each agent will be equipped with three network interface cards, one of which is a wireless interface. This interface is necessary in order to sniff and detect rogue access points. The following are the functions of agents:

1. Accept registration and unregistration messages for APs and client cards from the Central Administrator

2. Keep scanning the cell for rogue access points (Those access points that are not registered with the central administrator) and notify the concerned personnel if found

3. Detect promiscuous wireless nodes

4. Block unauthorized users

\section{How the System Works}

\subsection{Registration and Unregistration of APs}

Figure (2) shows the registration process of an access point. In order to register an access point, information such as MAC address of the access point is necessary. Apart from the MAC information, other information such as the cell in which the access point would be installed has to be chosen. Central Administrator forwards the MAC address to all the agents on the wireless backbone. Once the agents receive this information they will store the MAC address of the AP sent by the CA in their list of registered access points. Likewise, when an access point is unregistered, the central administrator will instruct all the agents to the delete the corresponding 
information from their lists. The reason that the CA sends the information to all the agents instead of just to the concerned agent is that, the cells might overlap and should there be an agent located in the overlapped area, that agent would consider the access point in the other cell as an unregistered one even though it is a registered access point.

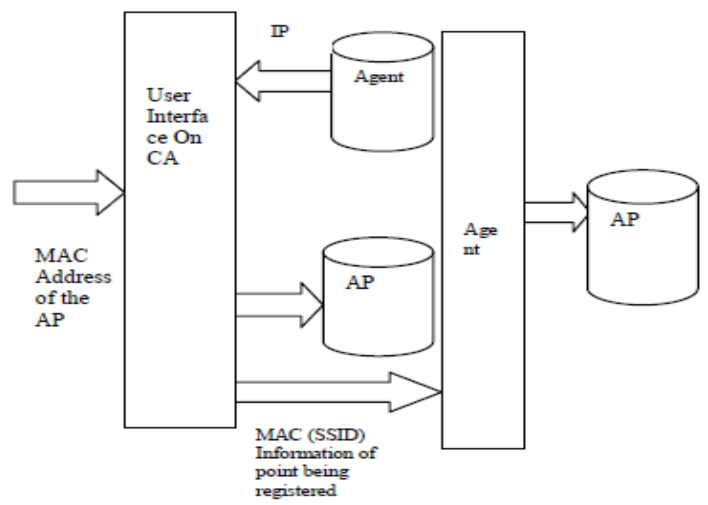

Figure (2): Access Point Registration Process

\subsection{Registration and Unregistration of Clients}

Figure (3) illustrates how the client cards are registered. In order to register a client, information such as user name, the user's department, user's e-mail address, and geographical information such as city and state and the MAC address of the user's wireless card is required. Once the information is submitted, an X.509 certificate will be emailed to the user of the card. This certificate can be used in implementing a secured tunnel between the user's mobile node and the central administrator. A copy of the user information is stored in a local flat file and sent to the agents. The agents upon receiving this information update their firewall policies, thus 
allowing the user to use the network resources. For unregistering a card, the central administrator instructs the agents to update their firewall policies to block the traffic from the user's wireless card.

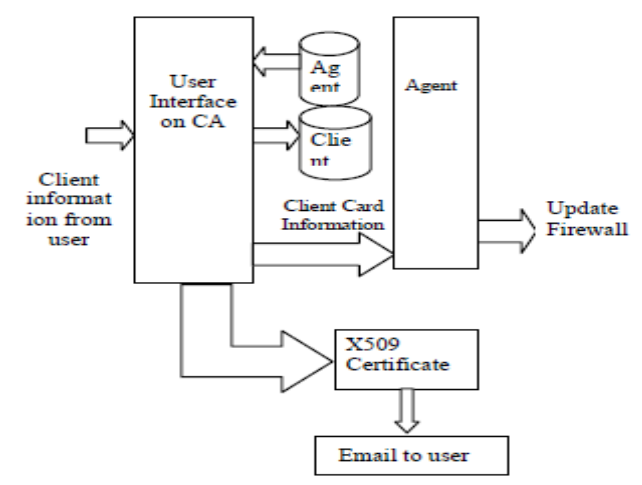

Figure (3): Client Registration Process

\subsection{Registration and Unregistration of Agents}

Figure (4) shows the agent registration process for registering agents. For registering agent information such as the name of the agent, the IP address of the agent, the cell in which the agent would be serving and the location information. Once the information corresponding to a particular agent is submitted, it will be stored in a local flat file and used when registering access points and client cards. Similarly when an agent is unregistered, the corresponding entry will be deleted from the list of agents.

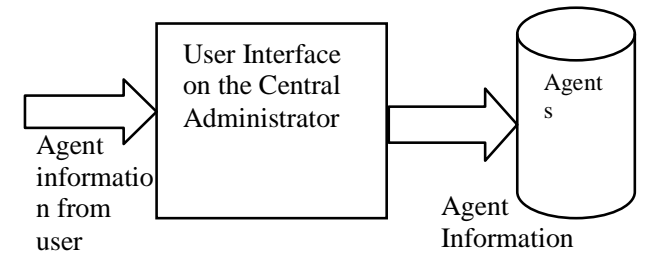

Figure (4): Agent Registration Process 


\subsection{Detection of Rogue Access points and Promiscuous nodes}

The following two figures (Figures 5 and 6) show the flowchart for detecting the rogue access points and the promiscuous nodes respectively.

Figure (5) shows how agents scan for rogue access points. The agents sniff for beacon frames using their wireless cards. When a beacon frame is found, they will check the SSID field (MAC address of the access point) of the frame with the existing MAC addresses in the list of registered access points and decide whether the access point in question is a legitimate or rogue one. Figure (6) shows how agents would detect promiscuous nodes in their respective cells. For each IP address in the subnet the agent is serving, the agent sends out a fake ARP request. Should the node with that IP address be in promiscuous node, the nodes? NIC would pass the ARP request to the kernel which in turn would send back an ARP response. The agent upon detecting this ARP response decides whether a given node is in promiscuous mode or not. If the agent already detected the node before it would go to the next IP in the subnet. On the other hand if the node is detected for the first time, the agent sends a warning message to the administrator and adds the node to its list of detected nodes. 


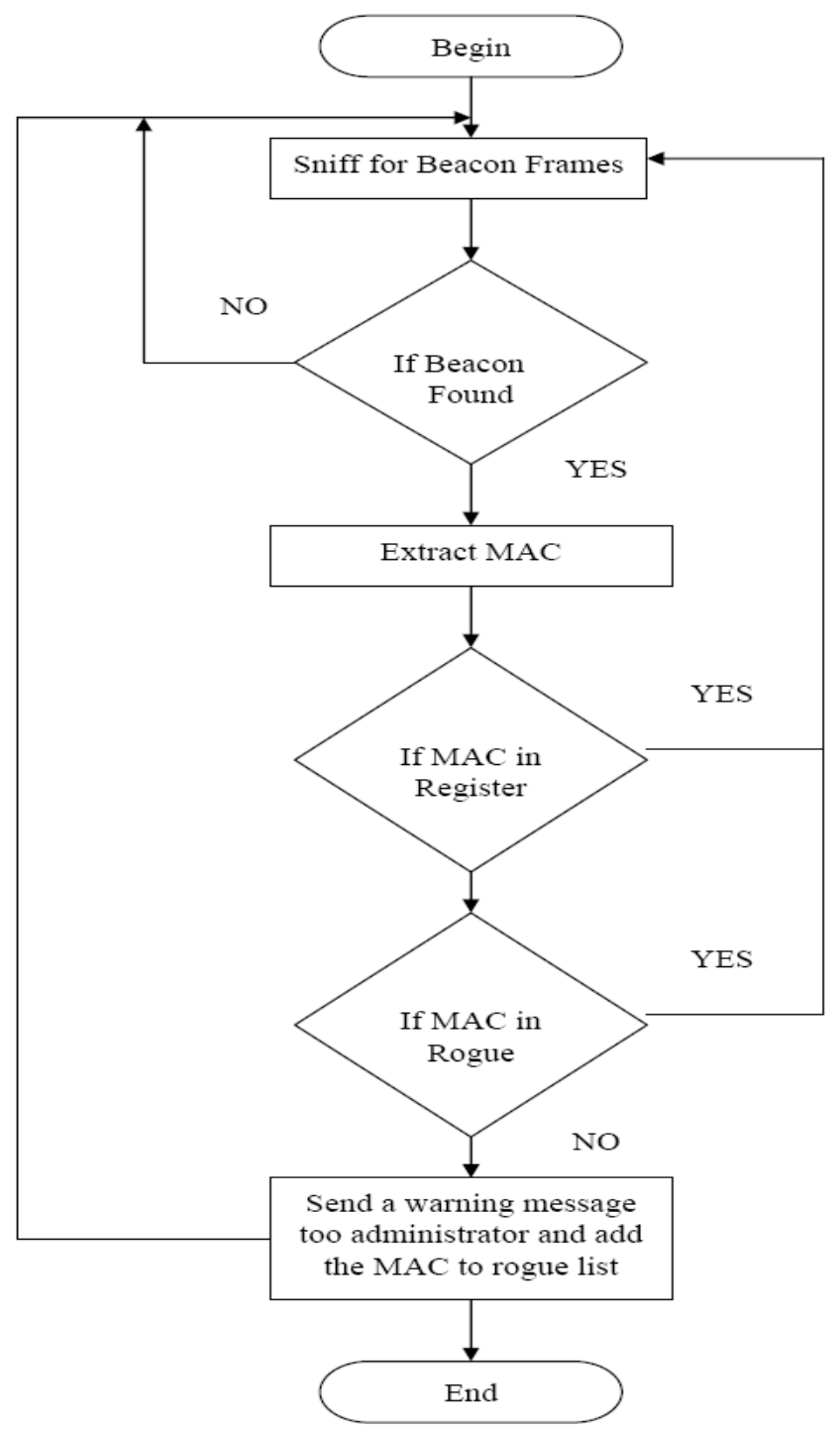

Figure (5): Flowchart for Detecting Rogue Access Point 


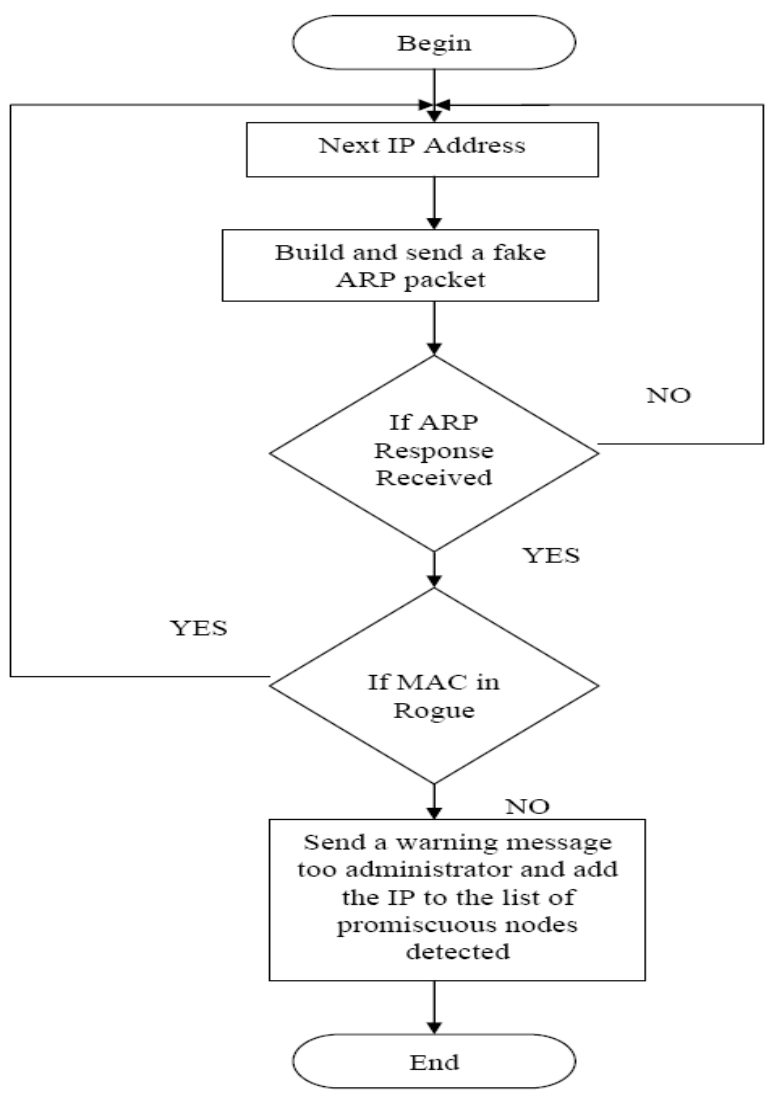

Figure (6): Procedure for Detecting Promiscuous Node

\section{Comparison with Existing System}

Nets tumbler [5] is a convenient tool that scans for insecure wireless network. It scans for the MAC address of access point, SID of the access point, signal strength, status of the WEP, the channels being used. It also plots SNR (Signal /Noise Ratio). The disadvantage of Nets tumbler is that rogue access points are not distinguished from legitimate access point.

APTools is a UNIX utility that queries ARP Tables and ContentAddressable Memory (CAM) for MAC Address ranges associated with 
802.11b Access Points in a Virtual LAN (VLAN). It utilizes Cisco Discovery Protocol (CDP) to propagate through a VLAN. This tool, however, is much focused and relies upon the Cisco products for propagation.

Kismet and stumbler are $802.11 \mathrm{~b}$ network sniffers and do almost the same job of sniffing for available wireless networks (or access points). Like APTools, Kismet can use CDP in a Cisco environment to propagate through a VLAN. Dstumbler is a Unix version of Netstumbler without the plotting facility. Airtraf [7] is a very sophisticated tool with a client server architecture that scans a network for access points. It is database software that mainly detects rogue access point across the organization and communicates back to a central server. This paper concentrates on providing extra features like detecting promiscuous nodes, blocking unauthorized users from accessing the network resources and providing X.509 certificates to the user of the wireless network.

\section{Conclusion}

The agent-based intrusion detection and response system is being developed to provide a secured wireless LAN solution. The main focus is on detecting unauthorized elements as soon as possible. By incorporating the feature to generate X.509 certificates, a VPN solution can be easily deployed to overcome the shortcomings of WEP because the three security goals namely data confidentiality, data integrity and access control are not delivered in WEP. The architecture proposed here can serve as a building block for finding efficient solutions for various other problems. In future, 
study of performance analysis on the agent and central administrator will be done.

\section{References}

[1]I.N.Borisov,I.Goldberg,D.Wagner, Intercepting Mobile Communications, In Mobicom, Rome, Italy, Jul. 2001.

[2] A. Stubblefield, J. Ioannidis, A.D. Rubin, Using the Fluhrer, Mantin, and Shamir Attack to break WEP, In Network and Distributed System Security Symposium. San Diego, California, Feb. 2006

[3] S. Fluhrer, I. Mantin, and A. Shamir., Weakness in the key scheduling algorithm of RC4, In Eigth Annual Workshop on selected Areas in Cryptography, Toronto, Canada, Aug. 2001.

[4] A. Mishra, W.A. Arbaugh, An Initial Security Analysis of the IEEE 802.1 Standard CS-TR- 4328, University of Maryland. http://www.cs.umd.edu/ waa/wireless.html. Accessed in Aug.2006

[5] Netstumbler, A windows tool to scan for available wireless networks, www.netstumbler.org. Accessed in Aug.2002

[6] Kismet, A linux tool used for scanning wireless networks, www.kismetwireless.net Accessed in Aug.2006

[7] AirTraf, A linux tool for gathering information about the available wireless networks, http://airtraf.sourceforge.net Accessed in Aug.2006 [8] 802.11Standard http://group.ieee.org/groups/802/11

[9] IPsec Security Architecture for the Internet protocol http://www.ietf.org/html.charters/ipsec-charter.html 